\title{
Discontinuing anticoagulation following successful atrial fibrillation ablation in patients with prior strokes
}

\author{
Roger A. Winkle • R. Hardwin Mead • Gregory Engel • \\ Melissa H. Kong • Rob A. Patrawala
}

Received: 14 July 2013 / Accepted: 27 August 2013 /Published online: 8 October 2013

(C) The Author(s) 2013. This article is published with open access at Springerlink.com

\begin{abstract}
Purpose This study was conducted to examine the outcomes in patients with prior stroke/transient ischemic attack (CVA/ TIA) after atrial fibrillation (AF) ablation and the feasibility of discontinuing oral anticoagulation (OAC).

Methods This study examined long-term outcomes following AF ablations in 108 patients with a history of prior thromboembolic CVA/TIA. Because of risks of OAC, we frequently discontinue $\mathrm{OAC}$ in these patients after successful ablation. These patients understand the risks/benefits of discontinuing $\mathrm{OAC}$ and remain on OAC for a longer time following successful AF ablation, compared to our patients without prior CVA/TIA.

Results Patient age was $66.2 \pm 9.0$ years with an average $\mathrm{CHADS}_{2}$ score $=3.0 \pm 0.9$ and $\mathrm{CHA}_{2} \mathrm{DS}_{2}$-VASc score $=4.1 \pm$ 1.4. Following 1.24 ablations, $71(65.7 \%)$ patients were AF free $2.8 \pm 1.6$ (median 2.3) years after their last ablation. OAC was discontinued in 55/71 (77.5\%) patients an average of 7.3 months following the final ablation. These 55 patients had $2.2 \pm 1.3$ (median 1.8)years of follow-up off of OAC. KaplanMeier analysis suggests little AF recurrence $>1$ year following initial or final ablations, suggesting that 1 year post successful ablation may be the appropriate time to consider discontinuing OAC. Thirty-seven patients had AF postablation, and 32/37 $(86.5 \%)$ remained on OAC. One patient with a mechanical
\end{abstract}

\footnotetext{
R. A. Winkle $(\bowtie) \cdot$ R. H. Mead · G. Engel • M. H. Kong •

R. A. Patrawala

Silicon Valley Cardiology, 1950 University Avenue, Suite 160

East Palo Alto, CA 94303, USA

e-mail: rawinkle@aol.com

R. A. Winkle · R. H. Mead · G. Engel • M. H. Kong •

R. A. Patrawala

Sequoia Hospital, Redwood City, CA, USA
}

valve had a stroke despite OAC. Bleeding occurred in $8.3 \%$ of patients on OAC and $0 \%$ of patients off OAC $(P=0.027)$. Conclusions Patients with prior CVA/TIAs, who undergo successful AF ablation, have a low incidence of subsequent thromboembolic events. Most patients who appear AF free postablation may be able to discontinue OAC after successful ablation with a low thromboembolic risk and with a reduced bleeding risk.

Keywords Atrial fibrillation · Ablation · Atrial fibrillation ablation · Anticoagulation after AF ablation

\section{Introduction}

Atrial fibrillation (AF) is a major cause of thromboembolic events including strokes (CVA) and transient ischemic attacks (TIAs) [1]. Radiofrequency ablation has the potential to eliminate $\mathrm{AF}$ in the majority of patients. Based on little objective data, current guidelines [1-3] recommend continuation of oral anticoagulation (OAC) with warfarin or newer anticoagulants after AF ablation in patients at high risk for thromboembolic events prior to ablation, even if the ablation appears to have eliminated AF. Despite the fact that AF ablation appears to reduce the risk for subsequent thromboembolic events in $\mathrm{AF}$ patients back to the level of stroke risk in patients with no history of AF across all CHADS2 profiles and ages [4], very few studies [5, 6] have described the outcome in high-risk patients with apparently successful AF ablation who have had OAC therapy discontinued. In the present study, we examined the long-term outcome in patients with a prior history of stroke/transient ischemic attack (CVA/TIA) who have undergone AF ablation. We especially emphasized the outcome in those patients with a successful AF ablation who have had OAC therapy discontinued. 


\section{Methods}

The subjects were 108 consecutive symptomatic patients undergoing AF ablation at Sequoia Hospital, Redwood City, California, from October 10, 2003, to December 31, 2011, who had a prior history of thromboembolic CVA/TIA. They were drawn from a total cohort of 1,411 patients undergoing $1,906 \mathrm{AF}$ ablations. Minimum follow-up was 1 year following final ablation. All signed written informed consent. Data collection was prospective and approved by the hospital IRB. AF type was categorized as paroxysmal (lasting $<1$ week or requiring pharmacological or electrical cardioversion in $<48 \mathrm{~h}$ ), persistent (lasting $>1$ week and $<1$ year or requiring pharmacological or electrical cardioversion between $48 \mathrm{~h}$ and 1 week), and longstanding persistent (lasting $>1$ year) [1] .

\subsection{Ablation protocol}

Our ablation protocol [7] and anticoagulation strategy [8] have been previously published. Antiarrhythmic drugs (AADs) were stopped $\geq 5$ half-lives and amiodarone $>3$ months before ablation. General anesthesia and right groin venous access were used in most ablations. A 7F duodecapolar catheter (St. Jude (St. Paul, MN) Livewire ${ }^{\mathrm{TM}}$ ) was placed around the tricuspid valve annulus with the distal poles in the coronary sinus. A 9F Boston Scientific (Natick, MA) Ultra Ice ${ }^{\mathrm{TM}}$ catheter guided the transseptal puncture, done using a $71-\mathrm{cm} \mathrm{St}$. Jude BRK ${ }^{\mathrm{TM}}$ or Baylis (Montreal, QC) NRG ${ }^{\mathrm{TM}}$ needle [9]. Patients had a femoral or radial arterial line. The St. Jude $\mathrm{NavX}^{\mathrm{TM}}$ mapping system was used in all cases. Prior to January, 2006, we used a closed-tip catheter (Boston Scientific Blazer IITM or Webster (Diamond Bar, CA) Celsius ${ }^{\mathrm{TM}} 8 \mathrm{~mm}$ ) and, thereafter, an open irrigated-tip catheter (Webster Thermocool ${ }^{\mathrm{TM}} 3.5 \mathrm{~mm}$, St. Jude Cool Path ${ }^{\mathrm{TM}}$, or Safire$\mathrm{Blu}^{\mathrm{TM}} 4.0 \mathrm{~mm}$ ). Most irrigated-tip catheter ablations were done using $50 \mathrm{~W}$ and the technique of "perpetual motion" where the catheter is moved back and forth across a small area and not left at single sites for extended times [10]. All patients underwent circumferential atrial ablation around all pulmonary veins (PVs) and a left atrium (LA) roof-line ablation. Only patients with right or LA isthmus flutter underwent a cavotricuspid or mitral isthmus linear ablation. Many patients had low posterior LA lines, and those with persistent AF and LA CFAE had them ablated. Some patients with longstanding persistent $\mathrm{AF}$ underwent ablation in the coronary sinus (at 30-35 W) and/or right atrium and superior vena cava isolation. After wide area ablation around all veins, a circumferential mapping catheter (7F Webster Lasso ${ }^{\mathrm{TM}}$ or St. Jude Reflexion Spiral ${ }^{\mathrm{TM}}$ ) was used to isolate all PVs defined as complete local electrical silence indicating entrance block. After May 2010, all patients underwent pacing from the PVs to document exit block. NavX $\mathrm{X}^{\mathrm{TM}}$ activation and entrainment mapping were used to ablate flutters and tachycardias.
Isoproterenol was given, and non-PV triggers were mapped and ablated.

\subsection{Anticoagulation}

Patients receiving warfarin continued it until 5 days preprocedure. Three days preprocedure, they began enoxaparin $1 \mathrm{mg} / \mathrm{kg}$ every $12 \mathrm{~h}$ with the last dose $24 \mathrm{~h}$ preablation. Patients on dabigatran had it discontinued $36 \mathrm{~h}$ preablation [10] or earlier based on renal function. Most patients underwent transesophageal echocardiogram. When the transseptal sheath entered the LA, we gave a heparin bolus followed by a 1,000-U/h infusion through the transseptal sheath adjusted by the activated clotting time (ACT). The initial target ACT was 300-350 s, reduced to 225 after the irrigated-tip catheter was introduced [8]. Postablation, we used enoxaparin $0.5 \mathrm{mg} / \mathrm{kg}$ q12h starting immediately postablation until warfarin (continued $\geq 3$ months) achieved an international normalized ratio (INR) of 2.0-3.0. After November 2010, patients receiving dabigatran following ablation [11] received two doses of enoxaparin $0.5 \mathrm{mg} / \mathrm{kg}$ - the first immediately after the procedure and a second dose $12 \mathrm{~h}$ later before resuming dabigatran the morning after the procedure approximately $10 \mathrm{~h}$ after the second dose of enoxaparin.

\subsection{Data collection and analysis}

For each patient, we recorded age, gender, AF duration and type, number of antiarrhythmic drugs failed, $\mathrm{CHADS}_{2}$ and $\mathrm{CHA}_{2} \mathrm{DS}_{2}-\mathrm{VAS}_{\mathrm{C}}$ scores, body mass index (BMI), LA size, prior cardioversions, and comorbidities including hypertension, diabetes, and coronary artery disease.

\subsection{Follow-up}

Many patients were treated with antiarrhythmic drugs during the 3-month blanking period postablation. Patients transmitted daily electrocardiogram (ECG) strips for 1-3 months postablation and were seen at 3 months when an echocardiogram and ambulatory ECG monitoring for 7-21 days were performed. Initial ablation failures, defined as recurrent AF, atrial tachycardia, or atrial flutter lasting $>30 \mathrm{~s}$ off of drugs $\geq 3$ months postprocedure were left on OAC, and the patient was encouraged to undergo a repeat ablation. Patients were seen or contacted frequently by phone from 3 to 12 months and returned for a 1-year follow-up with echocardiogram and 24-h ECG. Thereafter, every 6-12 months, patients were seen directly or contacted via telephone by research nurses or the attending physician, and arrhythmia records were obtained from hospitals and referring physicians. Patients with a prior CVA/TIA who were AF free postablation were continued on OAC longer than our usual time of 3 months in patients without a prior CVA/TIA. Prior to discontinuing OAC, 
CVA/TIA patients underwent a 7-21-day continuous recording with an ambulatory ECG monitor to document the absence of silent or asymptomatic AF. Pacemaker AF data were utilized when available. Patients were taught to check their pulse daily and to notify us immediately if they had any irregular pulse or symptoms suggesting a possible return of $\mathrm{AF}$, and ECG recorders were reissued to them.

\subsection{Long-term complications}

During the postprocedural follow-up, we evaluated all patients for CVAs/TIAs or systemic thromboembolism. We also evaluated each patient for bleeding complications. Since all patients initially started on OAC, we classified bleeding complications as to whether they occurred while a patient was on $\mathrm{OAC}$ or off OAC.

\subsection{Statistical analysis}

Statistical analysis was done using XLSTAT 2012 (Paris, France). Continuous data were described as mean \pm standard deviation and categorical data as counts and percentages. Student's $t$ test, chi-squared test, and Fisher's exact test were used to compare differences between clinical variables, the number of patients on $\mathrm{OAC}$ in the groups rendered AF free by ablation and those not rendered AF free, and the number of bleeding episodes among patients on OAC versus off OAC. Kaplan-Meier curves were generated for AF-free survival in years for the entire group by AF type for the initial ablation and for final outcome. All tests were two sided, and $P<0.05$ was considered statistically significant.

\section{Results}

\subsection{Patient population and ablation outcome}

The 108 patients with a history of CVA/TIA represented $7.9 \%$ of our patients undergoing AF ablation. Table 1 shows the clinical characteristics of these patients. The average age was $66.2 \pm 9.0$ years. LA size was $4.36 \pm 0.65 \mathrm{~cm}$. The average $\mathrm{CHADS}_{2}$ score was $3.0 \pm 0.9$, and CHADSVASC score was $4.1 \pm 1.4$. Forty $(37.0 \%)$ patients had paroxysmal AF, 50 (46.3\%) had persistent AF, and $18(16.7 \%)$ had longstanding persistent AF. Eighty-six patients underwent one ablation, 18 underwent two, and 4 underwent three ablations for an average of 1.24 ablations/patient. Seventy-one patients $(65.7 \%)$ were rendered AF free after one or more ablations, an average of 2.8 \pm 1.6 (median 2.3)years following their final ablation, and $37(34.3 \%)$ remained with AF. The number of prior cardioversions was the only statistically significant difference in the clinical characteristics between the patients who were rendered AF free by their ablation and those who were not. Table 2 shows the distribution of $\mathrm{CHADS}_{2}$ and $\mathrm{CHA}_{2} \mathrm{DS}_{2}$ VAS $_{\mathrm{C}}$ scores in the cohort of 108 patients.

\subsection{Long-term anticoagulation}

Among the 71 patients rendered AF free by ablation, 41 were on aspirin and 14 were on no anticoagulation. Thus, 55 of 71 patients $(77.5 \%)$ were off of OACs. Eight patients remained on warfarin, seven on dabigatran, and one on rivaroxaban. The reasons for continuation of OAC among these 16 patients were patient choice in three patients, physician choice in ten
Table 1 Clinical characteristics for all patients with prior CVA/ TIA, for the subgroups rendered $\mathrm{AF}$ free, and for those with recurrent $\mathrm{AF}$ postablation

${ }^{\text {a }}$ Statistically significant

\begin{tabular}{|c|c|c|c|c|}
\hline Clinical variable & $\begin{array}{l}\text { Entire group } \\
(n=108)\end{array}$ & $\begin{array}{l}\text { AF free postablation } \\
(n=71)\end{array}$ & $\begin{array}{l}\text { Recurrent AF } \\
\text { postablation }(n=37)\end{array}$ & $P$ value \\
\hline Left atrial size $(\mathrm{cm})$ & $4.36 \pm 0.65$ & $4.32 \pm 0.68$ & $4.49 \pm 0.65$ & 0.199 \\
\hline Age (years) & $66.2 \pm 9.0$ & $66.1 \pm 8.6$ & $66.4 \pm 9.1$ & 0.884 \\
\hline Duration of AF (years) & $7.8 \pm 7.1$ & $7.0 \pm 6.3$ & $9.0 \pm 8.2$ & 0.164 \\
\hline Repeat ablations & $20.4 \%$ & $20.5 \%$ & $21.1 \%$ & 1.000 \\
\hline$\%$ Male & $62.9 \%$ & $64.8 \%$ & $59.5 \%$ & 0.533 \\
\hline Prior cardioversions & $55.5 \%$ & $47.9 \%$ & $70.3 \%$ & $0.041^{\mathrm{a}}$ \\
\hline $\begin{array}{l}\text { Number of antiarrhythmic } \\
\text { drugs failed }\end{array}$ & $1.45 \pm 1.16$ & $1.38 \pm 1.22$ & $1.58 \pm 1.00$ & 0.386 \\
\hline Average $\mathrm{CHADS}_{2}$ score & $3.0 \pm 0.9$ & $3.1 \pm 0.9$ & $3.0 \pm 1.0$ & 0.772 \\
\hline Average $\mathrm{CHA}_{2} \mathrm{DS}_{2}$ - $\mathrm{VAS}_{\mathrm{C}}$ score & $4.1 \pm 1.4$ & $4.1 \pm 1.3$ & $4.0 \pm 1.6$ & 0.886 \\
\hline Hypertension & $62.9 \%$ & $69.0 \%$ & $51.3 \%$ & 0.093 \\
\hline Diabetes & $14.5 \%$ & $15.1 \%$ & $13.5 \%$ & 1.000 \\
\hline Coronary artery disease & $26.8 \%$ & $22.5 \%$ & $35.1 \%$ & 0.176 \\
\hline Body mass index & $28.6 \pm 5.0$ & $28.4 \pm 4.7$ & $29.0 \pm 5.7$ & 0.539 \\
\hline Paroxysmal AF & $37.0 \%$ & $43.6 \%$ & $24.2 \%$ & 0.060 \\
\hline Persistent AF & $46.3 \%$ & $43.6 \%$ & $53.8 \%$ & 0.310 \\
\hline Longstanding AF & $16.7 \%$ & $12.7 \%$ & $22.0 \%$ & 0.173 \\
\hline
\end{tabular}


Table 2 Number of patients by $\mathrm{CHADS}_{2}$ and $\mathrm{CHA}_{2} \mathrm{DS}_{2}-\mathrm{VAS}_{\mathrm{C}}$ scores

\begin{tabular}{lll}
\hline Score & $\begin{array}{l}\text { Number with each } \\
\mathrm{CHADS}_{2} \text { score }\end{array}$ & $\begin{array}{l}\text { Number with each } \\
\mathrm{CHA}_{2} \mathrm{DS}_{2}-\mathrm{VAS}_{\mathrm{C}} \text { score }\end{array}$ \\
\hline 2 & 33 & 18 \\
3 & 46 & 20 \\
4 & 20 & 28 \\
5 & 8 & 24 \\
6 & 1 & 13 \\
7 & na & 4 \\
8 & na & 1 \\
\hline
\end{tabular}

patients, patient and physician choice in two patients, and dilated cardiomyopathy in one patient. There were no clinical differences between those 55 patients stopping OAC and the 16 continuing on $\mathrm{OAC}$ with regard to age $(66.3 \pm 8.5$ vs. $66.0 \pm$ 9.2, $P=0.90), \mathrm{CHADS}_{2}$ score $(3.2 \pm 0.9$ vs. $3.1 \pm 0.7, P=1.0)$, or LA size ( $4.2 \pm 0.6$ vs. $4.3 \pm 0.7, P=0.70)$. Among the 37 patients with AF persisting following ablation, 3 were on aspirin, 2 were on no anticoagulants, 20 were on warfarin, 11 were on dabigatran, and 1 was on rivaroxaban. Of the five patients failing ablation and off of $\mathrm{OAC}$, three refused anticoagulation but were completely $\mathrm{AF}$ free on an antiarrhythmic drug, and two were off OAC because of recurrent bleeding. The difference in numbers of patients off of OAC in the AF-free $(55 / 71)$ versus AF recurrent (5/37) patients was statistically significant $(P<0.0005)$.

\subsection{Timing of anticoagulant discontinuation}

The average duration of follow-up after the patient's last AF ablation for the entire cohort of 108 patients was $2.8 \pm 1.6$ years

\section{AF Free Time after Initial Ablation by AF Type}
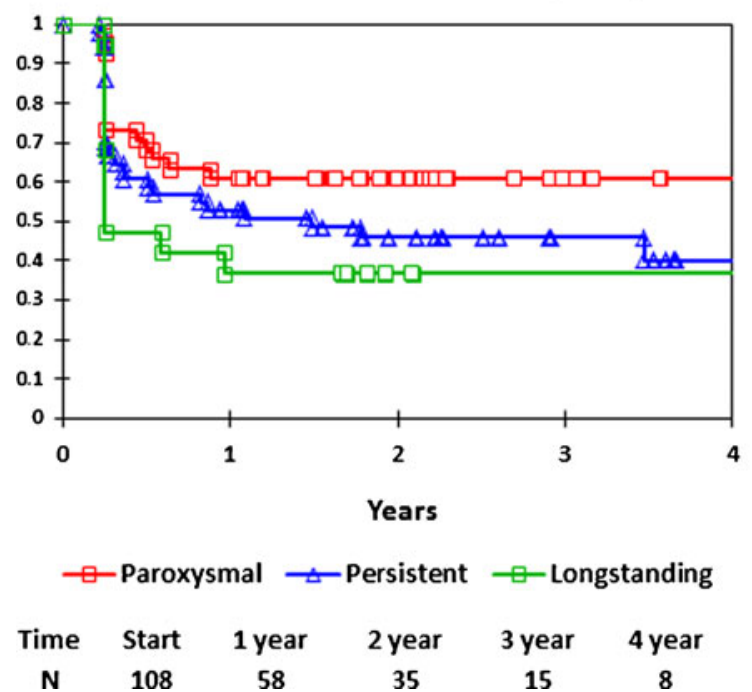

(median 2.3 years). All patients were followed for a minimum of 1 year following their final ablation. Among the 55 patients who appeared AF free following ablation and in whom $\mathrm{OAC}$ was discontinued, OAC was discontinued an average of 7.3 months following their final ablation. The duration of follow-up after discontinuation of OAC averaged $2.2 \pm 1.3$ (median $=1.8$ ) years.

\subsection{Time of AF recurrence following initial and final ablation}

Figure 1 shows the Kaplan-Meier AF-free curve for the entire cohort following initial and final ablations by AF type. It can be seen that for both the initial ablation and the final outcome, the more persistent the AF, the worse the outcome. The curve becomes nearly flat at 1 year after the initial ablation. Of the 58 patients who remained AF free for more than 1 year after initial ablation, there were only three $(5.2 \%)$ having a later recurrence of AF. After the final ablation, the curves also become nearly flat after approximately 1 year of follow-up. Following the final ablation, 72 patients were AF free at 1 year and only one (1.4\%) had a relapse after 1 year, and that patient relapsed at 1.1 years. Our data suggests one should wait for 1 year after an initial or repeat ablations before discontinuing OAC in patients with a prior CVA/TIA who appear to have a successful ablation.

\subsection{Clinical outcomes}

There were no CVA/TIA events or systemic thromboembolic events in any of the 71 patients who were rendered AF free following successful ablation. Among the 37 patients who remained with $\mathrm{AF}$, one patient with a mechanical heart valve had a thromboembolic event on warfarin despite a therapeutic

AF Free Time after Final Ablation by AF Type
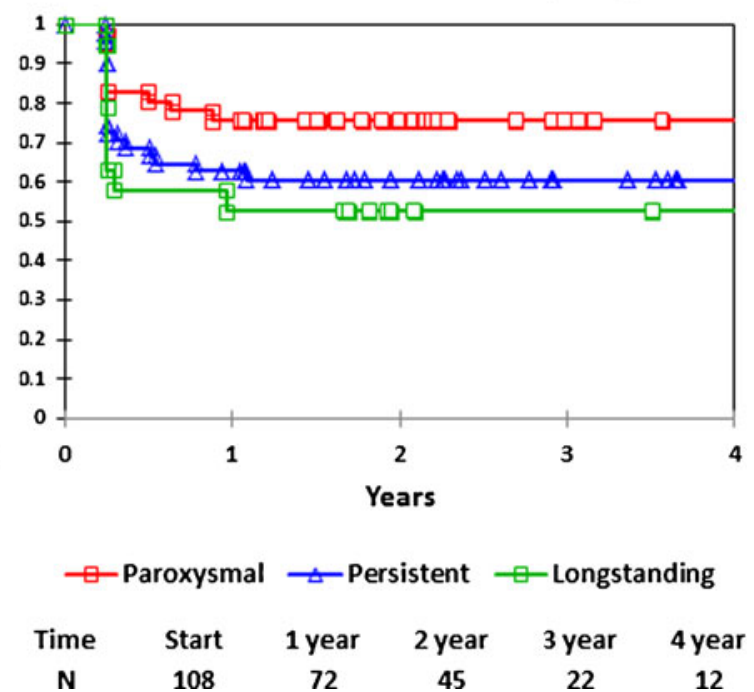

Fig. 1 Long-term AF-free rates by AF type (red=paroxysmal, blue=persistent, and green=longstanding persistent AF) for the initial ablation (left panel) and after final ablation (right panel) 
INR. There were nine $(8.3 \%)$ instances of bleeding (rectal bleeding in three, gastrointestinal bleeding in two, epistaxis in two, bleeding skin graft in one, and leg hematoma after a fall in one) among patients on OAC versus no bleeding events in the group in whom OAC was discontinued $(P=0.027)$. There were three late deaths, two due to progressive congestive heart failure in patients with preexisting cardiomyopathies and one due to cancer.

\section{Discussion}

Our study has several important findings. First, there is a low incidence of thromboembolic events in patients with a prior history of CVA/TIA who undergo successful AF ablation. Second, anticoagulation can be safely discontinued in the majority of patients who appear AF free following ablation with fewer bleeding complications in patients in whom OAC is discontinued. Third, these patients should be left on anticoagulation longer than the usual 3 months used for lowrisk patients following an ablation. Our data suggest that patients with a prior CVA/TIA who undergo ablation should be left on OAC for 1 year following a successful ablation to minimize the risk associated with $\mathrm{AF}$ recurrence.

All of the major guidelines [1-3] currently recommend continuing $\mathrm{OAC}$ in patients with significant risk factors for thromboembolic events following ablation regardless of the ablation outcome. These guideline recommendations are made on a paucity of actual clinical data. Bunch et al. [4] demonstrated that following AF ablation, patients have a lower rate of subsequent stroke across all ranges of $\mathrm{CHADS}_{2}$ scores. They showed that the risk of subsequent stroke in 4,212 consecutive AF ablation patients was lower than a matched control group of patients with AF who do not undergo ablation and was similar to a matched group without $A F$. What data are available (Table 3 ) suggest that it may be safe to discontinue anticoagulation in high-risk patients, including those with prior CVA/TIA, following an apparently successful $\mathrm{AF}$ ablation. Oral et al. evaluated 755 patients undergoing an AF ablation, 522 of whom were AF free following the ablation [5]. Warfarin was discontinued in $79 \%$ of 256 patients without risk factors for thromboembolic events. It was also discontinued in $68 \%$ of 266 patients with one or more risk factors for thromboembolic events. No patients in either group had a thromboembolic event with an average of $25 \pm$ 8.8 months of follow-up. In that study, 23/34 patients with a prior history of CVA/TIA were AF free. Among those 23 patients, ten stopped OAC and 13 remained on OAC. All 11 patients who continued to have $\mathrm{AF}$ remained on OAC. None of the 34 patients with a prior CVA/TIA had a thromboembolic event during follow-up.

Themistoclakis et al. published the pooled outcomes from five major ablation centers willing to stop OAC following successful ablation despite the published guideline recommendations [6]. They reported on 3,355 patients who were $\mathrm{AF}$ free following ablation. OAC was discontinued in 2,692 $(80.2 \%)$ patients. There were 594 patients with $\mathrm{CHADS}_{2}$ score $\geq 2$, and $347(58.4 \%)$ were off of OAC. There were 230 patients $(6.9 \%)$ with a prior history of CVA/TIA, and 125 of these (54.3\%) were off of anticoagulant therapy (Table 3 ). None of the patients with a history of prior CVA/TIA (on or off of OAC) experienced a thromboembolic event during follow-up. The ischemic stroke risk for patients in whom OAC was discontinued was $0.03 \% / y e a r$, and it was $0.23 \% /$ year for those patients on OAC. The major bleeding rate was $0.98 \%$ /year for those patients on OAC and $0.02 \%$ /year for those in whom it was discontinued $(P<0.0001)$.

Saad et al. examined the long-term risk of thromboembolic events off of OAC in 327 patients with a successful AF catheter ablation [12]. The CHADS 2 score was 2 or 3 in $68.8 \%$. Patients with higher $\mathrm{CHADS}_{2}$ scores had the time of OAC discontinuation extended from 3 to 6-12 months postablation. OAC was discontinued in $91 \%$ of these patients, none of whom had a thromboembolic event during follow-up. There were three $(0.9 \%)$ symptomatic hemorrhagic strokes in patients receiving OAC. Chao et al. examined the longterm adverse events (CVA, TIA, systemic embolism, pulmonary embolism, or death) in 565 patients undergoing AF ablation [13]. OAC was discontinued in some but not all high-risk patients. They found an adverse event rate of $2.8 \%$ among patients without $\mathrm{AF}$ versus $9.6 \%$ in those with AF recurrence $(P=0.001)$. Although both the $\mathrm{CHADS}_{2}$ and $\mathrm{CHA}_{2} \mathrm{DS}_{2}-\mathrm{VAS}_{\mathrm{C}}$ scores predicted adverse events, the authors did not examine outcomes separately for the patients who were AF free compared to those who continued to have AF.

Table 3 Summary of literature reports on stopping OAC in patients with prior CVA/TIA following a successful AF ablation

\begin{tabular}{lcccc}
\hline & $\begin{array}{l}\text { Total number of patients } \\
\text { AF free postablation }\end{array}$ & $\begin{array}{l}\text { Number of AF-free } \\
\text { patients off of OAC }\end{array}$ & $\begin{array}{l}\text { Subsequent thromboembolic } \\
\text { events }\end{array}$ & $\begin{array}{l}\text { Duration of follow-up } \\
\text { (months) }\end{array}$ \\
\hline Oral et al. [5] & 23 & $10(43 \%)$ & $0(0.0 \%)$ & $25 \pm 8.8$ \\
Themistoclakis et al. [6] & 230 & $125(54 \%)$ & $0(0.0 \%)$ & $28 \pm 13$ \\
Present study & 71 & $55(77.5 \%)$ & $0(0.0 \%)$ & $26 \pm 16$ \\
Total & 324 & $190(58.6 \%)$ & $0(0.0 \%)$ & \\
\hline
\end{tabular}


In our patient population, we discontinued $\mathrm{OAC}$ an average of 7.3 months following their final ablation. Prior to stopping $\mathrm{OAC}$, we held discussions with the patients regarding the risks and benefits of discontinuing OAC. They were advised that many physicians would recommend continuing OAC. Although we had no CVA/TIA in any patient in whom we stopped OAC, the Kaplan-Meier curves in Fig. 1 suggest it may have been prudent to continue OAC in these patients with a prior CVA/TIA who appear AF free postablation for 1 year before discontinuing OAC. It is possible that we could have safely stopped OAC in virtually all of our patients who appeared AF free postablation; however, we tried to accommodate patients' and referring physicians' wishes in making a decision to stop OAC.

Advocates of leaving patients at high risk for stroke on $\mathrm{OAC}$ often point to the potential for silent AF burden which could place the patient at risk for thromboembolic events. Previous studies in our patients [14] and at a number of other centers [15-18] suggest that the burden of only silent AF is low in patients who are followed closely and remain clinically AF free over the long term. The single study [19] demonstrating a high incidence of only asymptomatic AF following ablation had a high percentage of patients on antiarrhythmic drugs and $77 \%$ on beta-blockers postablation, which may have blunted patients' perceptions of recurrent AF. The TRENDS study [20] examined AF burden in patients with implanted devices in patients with and without a known history of paroxysmal AF. When patients experienced an episode of AF greater than $5.5 \mathrm{~h}$ in any 30 -day period, they had an increased risk of thromboembolic events. In a previous study in our cohort of AF ablation, patients were evaluated an average of 3 years postablation by 7-day monitoring and implanted devices [14]. Only 1 of 203 patients had a single episode of AF lasting longer than $5.5 \mathrm{~h}$. The patients in the TRENDS study did not undergo AF ablation and therefore would be expected to have a higher risk of subsequent CVA/ TIA [4] than the patients in our series who did undergo AF ablation. It is also likely that most of our highly symptomatic patients would have noticed a 5.5-h episode of AF and notified us of their relapse.

Our long-term outcomes in these patients were reasonable when examined by AF type (Fig. 1), but were slightly worse when compared with our entire cohort of patients undergoing AF ablation [7]. This lower AF-free rate may in part be due to the high $\mathrm{CHADS}_{2}$ scores in the present study. Chao et al. reported that for 247 patients with paroxysmal AF, the longterm $\mathrm{AF}$-free rate was $87 \%$ for those with a $\mathrm{CHADS}_{2}$ score of 0 and only $54.1 \%$ for $\mathrm{CHADS}_{2}$ scores of $\geq 3$ [21]. The Kaplan-Meier curves in the present group of patients with CVA/TIA appeared "flatter" with fewer late relapses than have been reported in many other follow-up studies including our own reports on our entire cohort of $\mathrm{AF}$ ablation patients [7]. It is possible that the more intense early EKG monitoring in the patients with previous CVA/TIA detected most of the ablation failures earlier postablation which resulted in a lower early success rate and a "flatter" late Kaplan-Meier curve.

\subsection{Limitations}

Our study is a single-center study with a relatively small number of patients and a follow-up of only slightly more than 2 years. Despite being a high-volume AF ablation center, we only had 108 patients undergoing ablation with prior CVA/ TIA. This suggests that cumulative data from multiple centers, such as shown in Table 3, will be required to definitively show the safety of discontinuing OAC. Patients for whom anticoagulation was discontinued were not randomized. However, given the extremely low rate of CVA/TIA in these patients postablation, it is unlikely enough patients could ever be recruited into a randomized trial to show a reduction in events by continuing OAC. The low rate of late recurrences in our study may have also contributed to the lack of late thromboembolic events after OAC was discontinued. It seems unlikely that we had a high rate of silent $\mathrm{AF}$ in these patients. If that were the case, one would have anticipated more thromboembolic events when OAC was discontinued. All of our patients were highly symptomatic before ablation and thus more likely to be aware of clinical AF failures postablation and to notify us immediately. Our findings should not be extrapolated to less symptomatic or asymptomatic patients undergoing $A F$ ablation. All patients with risk factors for CVA/TIA need lifelong follow-up after OAC is discontinued as they will always be at risk for AF recurrence. All of our patients were followed indefinitely by the ablating physicians, who continued to participate in anticoagulation decision making, in addition to their other physicians.

\section{Conclusions}

Patients with prior CVA/TIAs, who undergo successful AF ablation, have a low incidence of subsequent thromboembolic events. Most patients who appear AF free postablation may be able to discontinue OAC after successful ablation with a low thromboembolic risk and with a reduced bleeding risk. The absence of thromboembolic events in our patients with prior CVA/TIA and in other reported series of high-risk patients for whom OAC has been discontinued following an apparently successful AF ablation suggests that highly symptomatic patients, followed closely and indefinitely, may be able to safely consider discontinuation of OAC. Our results suggest the need for a multicenter randomized trial to definitively determine the risk benefit of continuing or stopping $\mathrm{OAC}$ in these patients after a successful AF ablation. 
Acknowledgments Patricia Barberini, R.N., Cynthia Lebsack, Pharm.D, and William Fleming and Glenda Rhodes assisted with data and manuscript management.

\section{Conflict of interest None}

Open Access This article is distributed under the terms of the Creative Commons Attribution License which permits any use, distribution, and reproduction in any medium, provided the original author(s) and the source are credited.

\section{References}

1. Calkins, H., Kuck, K. H., Cappato, R., Brugada, J., Camm, A. J., Chen, S., et al. (2012). 1012 HRS/EHRA/ECAS expert consensus statement on catheter and surgical ablation of atrial fibrillation: recommendations for patient selection, procedural techniques, patient management and follow-up, definitions, endpoints, and research trial design. Journal of Interventional Cardiac Electrophysiology, 33, 171-257.

2. Camm, A. J., Kirchhof, P., Lip, G. Y. H., Schotten, U., Savelieva, I., Ernst, S., et al. (2010). Guidelines for the management of atrial fibrillation. European Heart Journal, 31, 2369-2429.

3. Verma, A., Macle, L., Cox, J., \& Skanes, A. C. (2011). Canadian Cardiovascular Society atrial fibrillation guidelines 2010: catheter ablation for atrial fibrillation/atrial flutter. Canadian Journal of Cardiology, 27, 60-66.

4. Bunch, T. J., May, H. T., Blair, T. L., Weiss, J. P., Crandall, B. G., Osborn, J. S., et al. (2013). Atrial fibrillation patients have long-term stroke rates similar to patients without atrial fib regardless of CHADS2 score. Heart Rhythm., 10, 1272-1277.

5. Oral, H., Chugh, A., Özaydin, M., Good, E., Fortino, J., Sankaran, S., et al. (2006). Risk of thromboembolic events after percutaneous left atrial radiofrequency ablation of atrial fibrillation. Circulation, 114, 759-765.

6. Themistoclakis, S., Corrado, A., Marchlinski, F. E., Jais, P., Zado, E., Rossillo, A., et al. (2010). The risk of thromboembolism and need for oral anticoagulation after successful atrial fibrillation ablation. Journal of the American College of Cardiology, 55, 735-743.

7. Winkle, R. A., Mead, R. H., Engel, G., \& Patrawala, R. A. (2012). Trends in atrial fibrillation ablation: have we maximized the current paradigms? Journal of Interventional Cardiac Electrophysiology, 34, $115-123$.

8. Winkle, R. A., Mead, R. H., Engel, G., \& Patrawala, R. A. (2011). Safety of lower activated clotting times during atrial fibrillation ablation using open irrigated tip catheters and a single transseptal puncture. The American Journal of Cardiology, 107, 704-708.

9. Winkle, R. A., Mead, R. H., Engel, G., \& Patrawala, R. A. (2011). The use of a radiofrequency needle improves the safety and efficacy of transseptal puncture for atrial fibrillation ablation. Heart Rhythm, 8, 1411-1415.

10. Winkle, R. A., Mead, R. H., Engel, G., \& Patrawala, R. A. (2011). Atrial fibrillation ablation: perpetual motion of open irrigated tip catheters at 50 watts is safe and improves outcomes. Pacing and Clinical Electrophysiology, 34, 531-539.

11. Winkle, R. A., Mead, R. H., Engel, G., Kong, M. H., \& Patrawala, R. A. (2012). The use of dabigitran immediately following atrial fibrillation ablation. Journal of Cardiovascular Electrophysiology, 23, 264-268.

12. Saad, E. B., d'Avila, A., Costa, I. P., Aryana, A., Slater, C., Costa, R. E., Inácio, L. A., Jr., Maldonado, P., Neto, D. M., Camiletti, A., Camanho, L. E., \& Polanczyk, C. A. (2011). Very low risk of thromboembolic events in patients undergoing successful catheter ablation of atrial fibrillation with a $\mathrm{CHADS}_{2}$ score $\leq 3$. A long-term outcome study. Circulation. Arrhythmia and Electrophysiology, 4, 615-621.

13. Chao, T. F., Lin, Y. J., Tsao, H. M., Tasi, C. F., Lin, W. S., Chang, S. L., et al. (2011). CHADS 2 and $\mathrm{CHA}_{2} \mathrm{DS}_{2}$-VASc scores in the prediction of clinical outcomes in patients with atrial fibrillation after catheter ablation. Journal of the American College of Cardiology, $58,2380-2385$.

14. Winkle, R. A., Mead, R. H., Engel, G., Kong, M. H., \& Patrawala, R. A. (2012). Atrial arrhythmia burden on long-term monitoring in asymptomatic patients late after atrial fibrillation ablation. The American Journal of Cardiology, 110, 840-844.

15. Steven, D., Rostock, T., Lutomsky, B., Klemm, H., Servatius, H., Drewitz, I., et al. (2008). What is the real atrial fibrillation burden after catheter ablation of atrial fibrillation? A prospective rhythm analysis in pacemaker patients with continuous atrial monitoring. European Heart Journal, 29, 1037-1042.

16. Oral, H., Veerareddy, S., Good, E., Hall, B., Cheung, P., Tamirisa, K., et al. (2004). Prevalence of asymptomatic recurrences of atrial fibrillation after successful radiofrequency catheter ablation. Journal of Cardiovascular Electrophysiology, 15, 920-924.

17. Klemm, H. U., Ventura, R., Rostock, T., Brandstrup, B., Risius, T., Meinertz, T., et al. (2006). Correlation of symptoms to ECG diagnosis following atrial fibrillation ablation. Journal of Cardiovascular Electrophysiology, 17, 146-150.

18. Verma, A., Minor, S., Kilicaslan, F., Patel, D., Hao, S., Beheiry, S., et al. (2007). Incidence of atrial arrhythmias detected by permanent pacemakers (PPM) post-pulmonary vein antrum isolation (PVAI) for atrial fibrillation (AF): correlation with symptomatic recurrence. Journal of Cardiovascular Electrophysiology, 18, 601-606.

19. Hindricks, G., Piorkowski, C., Tanner, H., Kobza, R., Gerds-Li, J., Carbucicchio, C., et al. (2005). Perception of atrial fibrillation before and after radiofrequency catheter ablation. Circulation, 112, 307313.

20. Glotzer, T. V., Daoud, E. G., Wyse, G., Singer, D. E., Ezekowitz, M. D., Hilker, C., et al. (2009). The relationship between daily atrial tachyarrhythmia burden from implantable device diagnostics and stroke risk: the TRENDS study. Circulation Arrhythmia and Electrophysiology, 2, 474-480.

21. Chao, T. F., Cheng, C. C., Lin, W. S., Tsao, H. M., Lin, Y. J., Chang, S. L., et al. (2011). Associations among the CHADS(2) score, atrial substrate properties and outcome of catheter ablation in patients with paroxysmal atrial fibrillation. Heart Rhythm, 8, 1155-1159. 